\title{
ANÁLISE COMPARATIVA DA RESISTÊNCIA FLEXURAL DE DUAS RESINAS INDIRETAS
}

\section{Comparative analysis of flexural strength of two indirect resins}

\author{
Blanca Liliana Torres León ${ }^{a}$ \\ Emilena Xisto Limab \\ Wanessa Freitas Aras ${ }^{c}$ \\ Emily Vivianne Freitas da Silva ${ }^{\mathrm{d}}$ \\ Cecilia Andrade Moinhos ${ }^{\mathrm{e}}$ \\ Priscila Silva Queiroz
}

\section{RESUMO}

Objetivos: O sistema de resinas compostas indiretas constitui uma alternativa de tratamento estético com o objetivo de solucionar alguns dos problemas presentes nas restaurações das cerâmicas e das resinas convencionais. O presente estudo teve o objetivo de avaliar e comparar a resistência flexural, em três pontos, de dois materiais restauradores indiretos, Sinfony ${ }^{\circledR}$ (3M-ESPE) e Ceramage $^{\circledast}$ (Shofu). Materiais e Métodos: Foram confeccionados 12 corpos de prova de cada material com $15 \mathrm{~mm}$ de diâmetro e $2 \mathrm{~mm}$ de espessura. Decorridas 24 horas após a confecção, os discos foram submetidos ao teste de resistência flexural em três pontos em uma máquina de ensaio universal EMIC, a uma velocidade constante de $0,5 \mathrm{~mm} / \mathrm{min}$ e com célula de carga de $50 \mathrm{Kgf}$. Os dados foram avaliados estatisticamente pelo Teste $t$ de Student, ao nível de significância de 5\%. Resultados: A resina Ceramage apresentou a maior média (98,90 MPa) de resistência à flexão pelo método biaxial comparado ao grupo Sinfony (86,90 MPa), sendo esta diferença estatisticamente significante. Conclusão: Dentro das limitações deste estudo, podemos concluir que a resina Ceramage apresentou maior resistência flexural que a resina Sinfony, sendo uma excelente indicação de material restaurador indireto para o clínico.

Palavras-chave: Resistência. Resinas compostas. Estética.

\section{ABSTRACT}

Purpose: The system of indirect composite resins is an alternative esthetics treatment aiming to solve some of the problems present in the ceramic restorations and conventional resins. The aim of this study was to evaluate and compare the flexural strength in three points, of two indirect restorative materials, Sinfony ${ }^{\circledR}$ (3M-ESPE) and Ceramage ${ }^{\circledR}$ (Shofu). Materials and Methods: Twelve samples were made of each material with $15 \mathrm{~mm}$ diameter and $2 \mathrm{~mm}$ thick. After 24 hours of preparation, the discs were subjected to flexural strength test at three points in a universal testing machine EMIC, at constant speed of $0.5 \mathrm{~mm} / \mathrm{min}$ and load cell of $50 \mathrm{Kgf}$. The data were analyzed by Student $\mathrm{T}$ test, the significance level of $5 \%$. Results: The Ceramage had the highest average (98.90 MPa) of resistance to bending by the method biaxial compared to Sinfony (86.90 $\mathrm{MPa})$, the difference had being statistically significant. Conclusion: Within the limitations of this study, we conclude that the Ceramage showed higher values of flexural strength than Sinfony, being an excellent option of indirect restorative material for the clinician.

Keywords: Mechanical phenomena. Composite resins. Esthetics.

\footnotetext{
a Professora Adjunta, Departamento de Prótese Dentária da Universidade Federal da Bahia (UFBA), Salvador, Bahia, Brasil.

${ }^{\text {b }}$ Professora Adjunta, Departamento de Prótese Dentária da Universidade Federal da Bahia (UFBA), Salvador, Bahia, Brasil.

c Professora Assistente, Departamento de Dentística, Escola Bahiana de Medicina e Saúde Pública (UESB), Salvador, Bahia, Brasil.

d Aluna de doutorado, Departamento de Materiais Odontológicos e Prótese, Universidade Estadual Paulista (UNESP), Araçatuba, São Paulo, Brasil.

e Cirurgiã dentista, Escola Bahiana de Medicina e Saúde Pública (UESB), Salvador, Bahia, Brasil.

${ }^{\mathrm{f}}$ Cirurgiã dentista, Escola Bahiana de Medicina e Saúde Pública (UESB), Salvador, Bahia, Brasil.
}

Autor de correspondência: Blanca Liliana Torres León - E-mail: blalitole@hotmail.com 


\section{INTRODUÇÃO}

Na odontologia atual, a estética tem sido valorizada devido à alta exigência dos pacientes que almejam, não apenas um sorriso com características da dentição natural, mas também harmonioso. Assim, as restaurações indiretas livres de metal podem ser consideradas uma excelente alternativa de material restaurador estético, pois mimetizam a translucidez e a cor natural do dente ${ }^{1,2}$.

As cerâmicas dentárias feldspáticas foram empregadas durante os séculos XIX e XX, tendo como características a translucidez, estabilidade química, coeficiente de expansão térmica próxima ao da estrutura dentária, baixa condutividade, compatibilidade biológica e alta resistência à compressão $0^{3,4}$. Entretanto, desvantagens como, sua fragilidade e alta resistência à abrasão, levaram ao seu desuso. Além disso, os cimentos empregados na sua adesão eram friáveis e solúveis, além de interferir na cor e na estética das restaurações ${ }^{4,5}$.

A confecção de restaurações em cerâmicas livres de metal tornou-se possível graças ao surgimento da odontologia adesiva e de cerâmicas reforçadas com diversos cristais como alumina, leucita, dissilicato de lítio e zircônia ${ }^{6}$. Estas partículas cristalinas atuam como bloqueadores de fendas quando a cerâmica é submetida às tensões de tração, aumentando a resistência do material e tornando-as superior à feldspática convencional ${ }^{4,7}$.

Paralelamente ao desenvolvimento das cerâmicas, os sistemas de resinas compostas indiretas foram aperfeiçoados e introduzidos na Odontologia. Estes materiais apresentam alta densidade de partículas de cerâmicas, estabilidade de cor, facilidade na execução do reparo, proporção de desgaste similar ao esmalte e técnicas de confecção menos sensível quando comparada com as cerâmicas ${ }^{5,7,8,9}$.

Recentemente, foi introduzido no mercado, um novo sistema de resina híbrida indireta com alta carga de silicato de zircônio (Ceramage). Esta resina apresenta 73\% de cerâmica microparticulada em uma matriz polimérica, que fortalece sua estrutura homogênea e oferece a este sistema propriedades similares às da cerâmica para o uso em estruturas suportadas por metal ou sem metal, tanto em restaurações anteriores quanto em posteriore ${ }^{10,11}$. Já a resina indireta Sinfony consiste em um material micro híbrido de consistência fluida, contendo $50 \%$ de carga inorgânica ${ }^{12}$.

Alguns estudos científicos avaliaram a resistência flexural da resina Sinfony ${ }^{13,14,15}$. Porém, poucos estudos avaliaram essa propriedade na resina Ceramage ${ }^{16,17}$, não tendo sido observada na literatura a comparação da resistência flexural entre essas marcas de resina. Essas resinas foram previamente comparadas com relação à sua adesão à zircônia ${ }^{18}$ e resistência à microtração a liga de Ti-6Al-4V $\mathrm{V}^{19}$. Assim, o propósito deste estudo foi avaliar e comparar in vitro a resistência à fratura entre os sistemas restauradores indiretos (Sinfony e Ceramage) através do teste de resistência flexural pelo método biaxial.

\section{Materiais e mÉtodos}

Neste trabalho foram avaliados dois materiais restauradores estéticos de confecção indireta: Sinfony (3M ESPE, Seefeld, Alemanha), Ceramage (Shofu, Kyoto, Japão) (Tabela 1). Foram confeccionados 12 discos de cada material com $15 \mathrm{~mm}$ de diâmetro e $2 \pm 0,06 \mathrm{~mm}$ de espessura. 
Tabela 1: Material, número de lote, composição química e fabricante dos materiais restauradores indiretos.

\begin{tabular}{lccc}
\hline Material/No Lote & $\begin{array}{c}\text { Composição Química } \\
\text { Básica (") }\end{array}$ & \% Volume das Partículas & Fabricante \\
\hline $\begin{array}{l}\text { Ceramage/ } \\
070842\end{array}$ & $\begin{array}{c}\text { Resina composta } \\
\text { micro híbrida }\end{array}$ & Silicato de zircônio: 73 & Shofu, \\
Sinfony/ & Kyoto, Japão \\
344331 & Resina composta & Vidro de Estrôncio: 40 & 3M ESPE, \\
& $\begin{array}{c}\text { micro híbrida a base } \\
\text { de UDMA, livre de } \\
\text { Bis-GMA TEGMA }\end{array}$ & Sílica Pirogênica: 5 & Alemanha \\
\hline
\end{tabular}

*Fonte: Fabricante

Todos os discos foram preparados na cor A2 para dentina da escala Vita (Vita Zahnfabrik, Bad Säckingen, Alemanha), de acordo com as especificações dos respectivos fabricantes, a partir de uma matriz de aço inoxidável bipartida de $15 \mathrm{~mm}$ de diâmetro e $2 \mathrm{~mm}$ de espessura. As espessuras das amostras foram aferidas com um paquímetro digital (Starrett, Brasil) com precisão de $0,01 \mathrm{~mm}$.

Para a obtenção dos discos Ceramage, foi aplicada uma camada fina de isolante (Ceramage Sep, Shofu, Kyoto, Japão) na matriz de aço. Após 30 segundos, pequenos incrementos da resina foram inseridos no interior da matriz utilizando-se uma espátula de inserção (S.S White, Rio de Janeiro, Brasil). Os incrementos de resina foram polimerizados por 60 segundos no fotopolimerizador Strobolux (EDG, São Paulo, Brasil) totalizando 3 camadas de resina. Após a inserção da última camada, uma placa de vidro foi utilizada para a prensagem da resina, permitindo o escoamento do excesso do material. O disco foi removido da matriz, e então o Oxy-Barrier (Shofu, Kyoto, Japão) foi aplicado sobre o mesmo, visando evitar a formação de uma camada superficial com polimerização inibida pelo oxigênio. Posteriormente, a amostra foi levada ao fotopolimerizador Strobolux (EDG, São Paulo, Brasil) por 5 minutos para fotopolimerização final.

Já para a obtenção dos discos de Sinfony, a matriz bipartida foi isolada com o isolante de moldes fabricado pela 3M ESPE. A inserção da resina foi realizada em camadas, com auxílio de uma espátula de inserção. Cada camada foi polimerizada por 25 segundos com o aparelho de fotoativação Visio Alfa (3M ESPE, Seefeld, Alemanha).

Posteriormente à fotopolimerização inicial, o disco foi retirado da matriz e levado ao fotopolimerizador Visio Beta (3M ESPE, Seefeld, Alemanha) em um banho de luz em dois tempos, inicialmente por 1 minuto de luz e depois mais 14 minutos de luz com associação a vácuo.

Os excessos ao redor dos discos foram removidos com a utilização de uma politriz horizontal (Arotec APL-4; Arotec S.A., São Paulo, Brasil) com lixas de granulação decrescente $\left(220,320\right.$ e 600) (Norton ${ }^{\circledR}$, São Paulo, Brasil), visando a obtenção de uma superfície plana. Posteriormente, os corpos-de-prova foram armazenados em umidade relativa de 100\% por 24 horas, em estufa (Quimis, São Paulo, Brasil) sob a temperatura de $37^{\circ} \mathrm{C}$.

Após o período de armazenamento, os corpos de prova foram apoiados em um dispositivo de base metálica, que permite a fixação através de três esferas de 3,2 $\mathrm{mm}$ de diâmetro e equidistantes entre si, com $10 \mathrm{~mm}$ entre os centros das mesmas. 
Foi realizado o teste de resistência flexural, em uma máquina de ensaio universal (Modelo DL - 2000, EMIC, Paraná, Brasil) a uma velocidade constante de 0,5 mm/min e com célula de carga de $50 \mathrm{Kgf}$.

Após a fratura, os dados de resistência à flexão biaxial foram calculados a partir das seguintes fórmulas:

$$
\begin{aligned}
& \text { (1) } \cdot X=(1+) \ln (B / C)^{2}+[(1-) / 2](B / C)^{2} \\
& \text { (2). } Y=(1+)\left[1+\ln (A / C)^{2}\right]+(1-)(A / C)^{2}
\end{aligned}
$$$$
\text { (3). } S=-0,2387\left[P(X-Y) / d^{2}\right]
$$

Onde P é a carga de fratura, d é a espessura da amostra, v é o coeficiente de Poisson, A é o raio do círculo de suporte, B é o raio da ponta aplicadora de força e C é o raio do corpo de prova.

Os resultados foram submetidos ao Teste de t de Student para comparação múltipla das medias ao nível de significância de 5\%.

\section{Resultados}

Houve diferença estaticamente significativa entre os dois sistemas $(\mathrm{p}<0,05)$ quando comparadas as médias e desvio-padrão (SD) da resistência flexural dos dois materiais restauradores indiretos (Tabela 2). A média da resistência flexural foi maior do grupo Ceramage (93,90 MPa) que do grupo Sinfony (86,90 MPa).

Tabela 2: Médias, desvio padrão e Teste t de Student da resistência flexural (MPa) dos materiais restauradores indiretos

\begin{tabular}{lcc}
\hline Materiais Restauradores Indiretos & Resistência Flexural (MPa) & Teste t Student \\
\hline Ceramage & $93,90(7,75)$ & A \\
Sinfony & $86,90(9,06)$ & B \\
\hline
\end{tabular}

Médias com letras diferentes diferem entre si pelo Teste t de Student $(p<0,05)$.

\section{Discussão}

A resistência à fratura dos materiais odontológicos é um tema discutido em vários trabalhos de pesquisa, pois avalia a partir de testes laboratoriais a sua fragilidade. Uma das maneiras mais utilizadas para quantificar a resistência à fratura dos materiais restauradores é o teste de resistência à flexão, por ser um ensaio mecânico confiável e de fácil execução podendo ser de três ou quatro pontos ${ }^{10,20}$.

Neste estudo, foram avaliadas as resinas indiretas laboratoriais Sinfony e a Ceramage através da resistência flexural pelo método biaxial. Foi observado que a Ceramage apresentou os maiores valores de resistência à flexão do que o grupo da Sinfony. Foram observados que os valores de resistência flexural para o Ceramage e Sinfony foram de 93,90 MPa e 86,90 MPa, respectivamente (Tabela 2). Estas diferenças talvez tenham ocorrido em função da 
quantidade, porcentagem e tamanho de carga inorgânica, que aumentam as propriedades mecânicas da resina Ceramage $\mathrm{e}^{10,11,12}$.

A alta resistência à flexão é necessária para suportar as cargas mastigatórias, principalmente em situações de estresse sem que haja fratura ${ }^{21}$. Os compósitos odontológicos devem resistir às forças desenvolvidas durante a mastigação e a quantidade de partículas inorgânicas de um compósito está diretamente relacionada às suas propriedades mecânicas e a sua aplicabilidade clínica ${ }^{4,7}$. Em virtude das características dinâmicas, e as verdadeiras tensões durante a mastigação são difíceis de serem quantificadas. A força mastigatória média mais alta possível de ser mantida é de aproximadamente $756 \mathrm{~N}^{4,22}$.

Alguns estudos científicos avaliaram a resistência flexural da resina Sinfony. Souza et al. ${ }^{14}$ avaliaram 3 marcas de resinas indiretas (VITA VMLC, Resilab e Sinfony) quanto à resistência à flexão após 24 horas de imersão em água destilada. Os autores não verificaram diferença estatística entre a resina Sinfony $(177,11 \mathrm{MPa})$ e as demais marcas. Já Göhring et al. ${ }^{13}$ avaliaram a resistência à flexão de 5 marcas de resinas indiretas (Sinfony, Belleglass, Sculpture, SR Adoro e Targis) e 1 direta (Tetric Ceram) e a influência do armazenamento em água e termociclagem nessa propriedade. Os autores observaram menores valores de resistência da resina Sinfony após 14 dias de imersão em água à $37^{\circ} \mathrm{C}(87,1 \mathrm{MPa})$ e termociclagem $(83 \mathrm{MPa})$ quando comparado às demais marcas. Por outro lado, Borges et al..$^{15}$ compararam 8 marcas comerciais (Sinfony, Signum, Solidex, Resilab, Adoro, Admira, Filtek Supreme e Venus) de resinas indiretas quanto à resistencia flexural. A resina Sinfony apresentou o maior valor de resistência (139,61 MPa), exceto da Filtek Supreme (149,12 MPa).

Com relação à resina Ceramage, Muñoz et al. ${ }^{16}$ a compararam com as resinas Filtek P60, Adoro e IPS e.max, com relação à resistência flexural. A resina Ceramage $(137,01 \mathrm{MPa})$ apresentou diferença estatística da resina Filtek P60 (157,98 MPa). Já LIM et al. (2016) compararam Ceramage, Z100, Lava Ultimate e Vita Enamic, verificando alto valor de resistência flexural da resina Ceramage (200 MPa), com diferença estatística da resina Vita Enamic (129 MPa).

Tendo por base os resultados obtidos nessa pesquisa, podemos indicar o Ceramage como material restaurador indireto. No entanto maiores pesquisas laboratoriais ou clínicos serão necessárias a fim de analisar a longevidade do material.

\section{ConclusÃo}

Com base nos resultados analisados e discutidos neste trabalho, foi possível concluir que, a resistência à flexão pelo método biaxial foi maior para o material restaurador Ceramage do que a Sinfony. Dessa forma, podemos atestar que a resina Ceramage é uma excelente opção de material restaurador indireto para o clínico.

\section{REFERÊNCIAS}

1. Miranda CB, Pagani C, Bottino MC, Benetti AR. A comparison of microhardness of indirect composite restorative materials. J Appl Oral Sci. 2003; 11(2):157-61.

2. Peutzfeldt A, Sahafi A, Flury S. Bonding of restorative materials to dentin with various luting agents. Oper Dent. 2011;36(3):266-73.

3. Rueda AO, Anglada M, Jimenez-Pique E. Contact fatigue of veneer feldspathic porcelain in dental zirconia. Dent Mater. 2015;31(3):217-24

4. Anusavice K], Shen C, Rawls HR. Phillips' science of dental materials. 12th ed. St. Louis:MO: Elsevier; 2012, 474-98.

5. Kakaboura A, Rahiotis C, Zinelis S, Al-Dhamadi YA, Silikas N, Watts DC. In vitro characterization of two laboratory-processed resin composites. Dent Mater. 2003;19(5):393-8. 
6. Wildgoose DG, Johnson A, Winstanley RB. Glass/ceramic/refractory techniques, their development and introduction into dentistry: A historical literature review. ] Prosthet Dent. 2004;91(2):136-43.

7. Prakki A, Cilli R, de Araújo PA, Navarro MF, Mondelli ], Mondelli RF. Effect of toothbrushing abrasion on weight and surface roughness of $\mathrm{pH}$-cycled resin cements and indirect restorative materials. Quintessence Int. 2007;38(9):e544-54.

8. Bottino MA, Giannini V, Miyashita E, Quintas AF. Metal free-Estética em Reabilitação Oral. 1 ed. São Paulo: Artes Médicas; 2001.

9. Manhart ], Chen HY, Mehl A, Hickel R. Clinical study of indirect composite resin inlays in posterior stress-bearing preparations placed by dental students: Results after 6 months and 1, 2, and 3 years. Quintessence Int. 2010;4(5):399-410.

10. Fernandez IM, Mogollones RF, Castilla MV. Flexural resistance of esthetic materials used by indirect restoration: a comparative in vitro study. Int ] Odontostomatol. 2013;7(2):315-8.

11. Ellakwa A, Martin FE, Klineberg I. Influence of implant abutment angulations and two types of fibers on the fracture resistance of ceramage single crowns. J Prosthodont. 2012;21(5):378-84.

12. Borba M, Della Bona A, Cecchetti D. Flexural strength and hardness of direct and indirect composites. Braz Oral Res. 2009;23(1):5-10.

13. Göhring TN, Gallo L, Lüthy H. Effect of water storage, thermocycling, the incorporation and site of placement of glass-fibers on the flexural strength of veneering composite. Dent Mater. 2005;21(8):761-72.

14. Souza ROA, Mesquita AMM, Pavanelli CA, Nishioka RS, Bottino MA. Avaliação da resistência à flexão de três resinas compostas de uso laboratorial. Int ] Dent. 2005;4(2):50-4.

15. Borges ALS, Borges AB, Barcellos DC, Saavedra CSFA, Paes Junior T]A, Rode SM. Avaliação da resistência flexural e módulo de elasticidade de diferentes resinas compostas indiretas. RPF Rev Pós Grad. 2012;19(2):50-6.

16. Muñoz IM, Florio MR, Velásquez CM. Flexural resistance of esthetic materials used by indirect restoration. A comparative in vitro study. Int J Odontostomat. 2013;7(2):315-8.

17. Lim K, Yap AU, Agarwalla SV, Tan KB, Rosa V.. Reliability, failure probability, and strength of resin-based materials for CAD/CAM restorations. ] Appl Oral Sci. 2016;24(5):447-52.

18. Van Vuuren W], Van Vuuren L], Torr B, Waddell ]N. Adhesion between zirconia and indirect composite resin. Int ] Adhes Adhes. 2016;69(C):72-8.

19. Fernandes CA, Ribeiro JC, Larson BS, Bonfante EA, Silva NR, Suzuki M, et al. Microtensile bond strength of resin -based composites to Ti-6Al-4V. Dent Mater. 2009;25(5):655-61.

20. Anusavice K]. Degradability of dental ceramics. Adv Dent Res. 1992;6:82-9.

21. Brandt WC, Cardoso L, Moraes RR, Correr-Sobrinho L, Sinhoreti MAC. Influence of light-curing units on the flexural strength and flexural modulus of different resin composites. Braz ] Oral Sci. 2008;7:1555-8.

22. Pennafort Junior LCC, Oliveira Junior SC, Deus EP. Systems analysis of denture fixed with four implants. Int ] Mech Eng Autom. 2014;1(4):229-35. 